\title{
Desigualdad, desarrollo humano y la consolidación urbano-regional en México
}

\begin{abstract}
Manuel Suárez. Universidad Nacional Autónoma de México, Ciudad de México, México. Naxbelli Ruiz. Universidad Nacional Autónoma de México, Ciudad de México, México. Javier Delgado. Universidad Nacional Autónoma de México, Ciudad de México, México.
\end{abstract}

RESUMEN | En este artículo se explora la asociación espacial entre el desarrollo humano, la consolidación urbana y la desigualdad en la distribución del ingreso en México. Dicha asociación se mide tanto de manera no paramétrica $(\lambda)$ como mediante un modelo de regresión geográficamente ponderada. Los resultados comprueban una mayor correlación entre la urbanización y la generación de condiciones de menor desigualdad que entre esta última y el desarrollo humano. Asimismo, muestran una influencia significativa del factor espacial (la distribución regional de la urbanización y el desarrollo humano) sobre los niveles de desigualdad municipal.

PALABRAS CLAVE | Desigualdad social, pobreza, urbanización

ABSTRACT | The article explores the spatial association between the Human Development Index, the Gini coefficient and the level of urbanization in Mexico. We determine the association between the three indexes with a non-parametric measure $(\lambda)$ as well as with a geographically weighted regression model. We find a negative association between urbanization and inequality, but mostly, our analysis suggests a strong importance of space (the regional distribution of the urban condition) in the local measures of inequality.

KEY WORDS | Social inequality, poverty, urbanization

Recibido el 8 de marzo de 2011, aprobado el 10 de noviembre de 2011

E-mail: Manuel Suárez, msuarez@igg.unam.mx | Naxhelli Ruiz, naxhelli.ruiz@googlemail.com | Javier Delgado, jdelgado@igg.unam.mx 


\section{Introducción}

El objetivo de este artículo es analizar la relación entre los patrones espaciales de la distribución de la desigualdad, el desarrollo humano y la urbanización en México, bajo el supuesto de que este último factor es el que más incide en la distribución de satisfactores clave para el consumo, el empleo y el ingreso, así como en la capacidad para acceder a ellos. En particular, se busca conocer hasta qué punto existe una asociación significativa entre los patrones de distribución de la desigualdad en el ingreso y las características del espacio geográfico. Para ello se compara el comportamiento espacial de tres indicadores asociados con el desarrollo social: el Índice de Desarrollo Humano (IDH), el índice de Gini con base en el ingreso, y el Índice de Consolidación Urbano-Regional (ICUR).

El Índice de Desarrollo Humano mide el logro promedio de un colectivo en tres dimensiones básicas del desarrollo social: la salud (esperanza de vida al nacer), la educación, y el ingreso como indicador de la privación de un estándar de vida aceptable (producto interno bruto per cápita) (United Nations Development Programme [UNDP] 2007, p. 355). Para la medición de la desigualdad se emplea un coeficiente de Gini territorial que mide la disparidad en el ingreso medio per cápita para cada territorio (Economic Commission for Latin America and the Caribbean [ECLAC], 2010, p. 126). Finalmente, para medir el grado de urbanización, utilizamos el Índice de Consolidación Urbano-Regional (ICUR) propuesto por Delgado, Larralde y Ansaldo (1999) y revisado por Galindo (2007) y Delgado, Galindo y Ricárdez (2008).

A partir de la descripción de la distribución nacional de esos índices, medimos la autocorrelación espacial entre ellos mediante la I de Moran (Moran, 1950) e identificamos la formación de conglomerados espaciales de cada uno mediante la I de Moran local (Anselin, 1995). Esos conglomerados se comparan mediante tablas de contingencia. Por último, corremos dos análisis de regresión para identificar la influencia del espacio geográfico sobre la desigualdad en la distribución del ingreso. Los parámetros de un primer modelo lineal, cuyos residuos muestran autocorrelación espacial, se comparan con un segundo modelo de regresión geográficamente ponderada. Esta comparación permite identificar la influencia parcial del espacio regional y el grado de consolidación urbana en la desigual distribución del ingreso.

El resultado muestra que, como indicador global de consolidación urbana, el ICUR explica dos veces más la variación en la distribución del ingreso que el IDH como indicador general de desarrollo humano. Ello demuestra una asociación positiva entre la urbanización y la generación de condiciones de menor desigualdad. El hecho de que el modelo econométrico-espacial sea más robusto que el modelo de regresión simple sugiere una importancia significativa de la espacialidad en la distribución de la desigualdad y la necesidad de caracterizarla no solo a partir del comportamiento de los hogares e individuos en una determinada unidad territorial, sino a partir de las asociaciones espaciales entre esas unidades con relación a su contexto regional. 
El artículo está dividido en cinco secciones. En la primera se identifica y discute la literatura relevante para comprender el problema en estudio. En la segunda se presenta la distribución geográfica de los diferentes índices y se muestran características socioeconómicas, agregadas para el país y para grupos de municipios, con diferentes niveles de pobreza y desigualdad. La tercera sección explica, en detalle, la metodología empleada. La cuarta muestra los resultados de los diversos análisis y da paso a una quinta sección de conclusiones.

\section{Revisión de la literatura}

Las discusiones sobre desigualdad y territorio en México se han centrado, casi exclusivamente, en mostrar la distribución espacial de las características de los hogares y de ciertas condiciones clave para el desarrollo humano.

En las propuestas más conocidas se relacionan el ingreso, educación, condiciones de la vivienda y tipo de ocupación, por medio de índices como el de marginación (Consejo Nacional de Población [Conapo], 2006) o el de rezago social (Consejo Nacional de Evaluación de la Política de Desarrollo Social [Coneval], 2007). Por su parte, Székely, López-Calva, Meléndez, Rascón y Rodríguez-Chamussy (2007), al analizar la relación entre pobreza y desigualdad con su distribución territorial, correlacionaron indicadores de pobreza (la línea de ingreso que marca el nivel de pobreza alimentaria) y desigualdad en el ingreso (Índice de Theil). En términos espaciales, los autores enfatizaron el peso de la desigualdad (al interior de las unidades territoriales, de estados y municipios) sobre la determinación de la desigualdad nacional (por encima de las diferencias entre unidades territoriales). A partir de ahí, discutieron la importancia de reducir las desigualdades de ingreso a nivel local como una estrategia de intervención en políticas públicas, sin abordar la dimensión regional del problema.

En general, las mediciones multidimensionales de la pobreza ${ }^{1}$ en México han combinado la medición de desigualdad y pobreza de forma complementaria, pero no han incluido explícitamente el componente espacial, excepto en términos de la condición administrativa urbana o rural de los estados y municipios.

La interrogante que surge es: ¿cuáles son los factores que determinan la dinámica espacial de la desigualdad, más allá de la distribución municipal y regional de los indicadores? Para responderla es necesario espacializar la explicación del porqué se generan dichas desigualdades. Si bien la desigualdad ha sido estudiada y discutida ampliamente desde puntos de vista tanto económicos como sociológicos (Atkinson, 1970; Sen, 1992; Kanbur, 2005), la discusión teórico-metodológica de la espacialidad y la distribución de la desigualdad en unidades espaciales ha sido menos explorada. ${ }^{2}$ Más limitado aún ha sido el estudio del papel del espacio en las condiciones que generan los procesos de desigualdad. ${ }^{3}$

\footnotetext{
Véase, por ejemplo, la revisión de los métodos multidimensionales en Boltvinik (2004, p. 446).

2 Una excepción es el trabajo de Getis, Mur y Zoller (2004), compilación de estudios de econometría espacial que abarca diversos temas y propuestas metodológicas, desde la propuesta de técnicas alternativas para la identificación de clusters espaciales hasta la discusión de mecanismos de inferencia sobre el comportamiento de la relación entre unidades espaciales en series temporales, pasando por técnicas para reducir el sesgo de las diferentes estrategias de agrupación y escalamiento.

3 Para una revisión de los enfoques de desigualdad espacial, véase Alasia (2002).
} 
En los años noventa se publicaron numerosos trabajos en economía abocados a estudiar los procesos de convergencia regional, ${ }^{4}$ que mostrarían las condiciones necesarias para que un conjunto de unidades territoriales tuviera un comportamiento similar en dimensiones específicas, por ejemplo, en cuanto al ingreso (Quah, 1996; Sala-i-Martin, 1996; Rey \& Montouri, 1999). Sin embargo, era evidente la necesidad de considerar también dimensiones como la polarización, concentración y estratificación social, o los problemas de agregación de datos, para interpretar y validar mejor las múltiples inferencias respecto de las desigualdades regionales (Alasia, 2002; Rey \& Janikas, 2005; Sastré-Gutiérrez \& Rey, 2008).

En ese sentido, la mayoría de los métodos de medición se ha centrado en la desigualdad entre individuos, y entre y al interior de grupos o clases (Cortés, 2002). Sin embargo, aún no se han resuelto las distintas interrogantes sobre la causalidad de las diferencias entre unidades espaciales o entre individuos distribuidos en un mismo espacio. En particular, una de las preguntas más importantes es saber si la variación del ingreso se explica mayormente por la distribución espacial de sus miembros en distintas unidades territoriales o si responde a la desigualdad individual o entre grupos sociales (casta, etnia, clase). Siendo así, su distribución espacial sería una expresión secundaria, pero no una explicación significativa. En términos de política social, la respuesta que demos a ello es fundamental para reducir la desigualdad y la pobreza, al decidir hacia dónde y a quiénes dirigir mayores recursos para el desarrollo social (Seth, 2009, p. 2).

Al debatir sobre el peso de la dimensión espacial como factor explicativo de la desigualdad, Kanbur y Venables (2005) han argumentado que la "mayor parte de las variaciones entre individuos se da dentro de las unidades espaciales, no entre individuos de distintas unidades espaciales, aun para desagregados muy finos” (p. 9). Según sus estimaciones, "el determinante clave del bienestar de los hogares en una región, por encima y ante las características específicas de los hogares, es la cantidad y calidad de la infraestructura de una región" (Kanbur \& Venables, 2005, p. 9; véase también Escobal \& Torero, 2005, p. 106).

A partir de esta premisa, es necesario explorar cuáles son los factores espaciales que generan la desigualdad, en el entendido de que esta no se deriva exclusivamente de las condiciones socioeconómicas individuales o de los hogares. Es decir, la descripción de la desigualdad de su distribución, a distintas escalas y agrupamientos, es insuficiente para explicarla. Además, cobran relevancia otras características del espacio geográfico que inciden directamente en la existencia de satisfactores y en las probabilidades de acceder a ellos. Ello implica que aparte de estudiar la distribución de características y recursos de individuos, hogares y/o localidades (hasta ahora, las unidades básicas de medición de la desigualdad), es necesario determinar los atributos explícitamente espaciales de las unidades geográficas donde aquellos se localizan. En particular, nos referimos a los recursos e infraestructuras que for-

4 La convergencia regional se refiere a los factores que inciden en la forma y velocidad con que unidades territoriales con menores niveles de ingreso (entendidas como proxy de un determinado estándar de vida) 'alcanzan' a aquellas que muestran mayores niveles, en un determinado lapso temporal, reduciendo así las disparidades existentes entre ambas. 
man la base material de los servicios de salud, educativos, del transporte y de uso de los espacios públicos, deportivos y culturales. Estos recursos son diferentes de los conjuntos de satisfactores (endowments) de los hogares y de los individuos y tienen la capacidad de funcionar como elementos para el bienestar. La razón para enfocarse en ellos es su influencia determinante sobre la accesibilidad a bienes y servicios, así como sobre los perfiles de consumo, el empleo y el ingreso. ${ }^{5}$ Es decir, hogares e individuos con niveles similares de satisfactores (como el ingreso) tienen potenciales diferentes, de acuerdo con las características de su entorno geográfico. ${ }^{6}$

En ese sentido, las diferencias sugeridas por Ziccardi (2008) entre la pobreza rural (que define como alimentaria) y la urbana (carencias patrimoniales derivadas del acceso diferencial a una vivienda digna, infraestructura y servicios básicos) dan pistas sobre el papel crucial que tienen los atributos geográficos para explicar la relación entre carencia y desigualdad. Se trata, en suma, de un enfoque espacial distinto a los que se basan en indicadores sobre la disponibilidad de bienes a nivel del hogar, así como a los índices de marginación y rezago social.

Siguiendo esa línea, en el presente artículo se propone una exploración de la desigualdad espacial que va más allá de la distribución de atributos, e incorpora el uso y características materiales del espacio como componentes esenciales de las diferencias sociales. Proponemos para ello explorar las características generales del espacio geográfico (a escala nacional) y su influencia sobre la desigualdad, mediante indicadores asociados con la urbanización que reflejen la concentración de bienes y servicios clave en el espacio. La propuesta del Índice de Consolidación Urbano-Regional (ICUR) (Delgado et al., 1999; Galindo, 2007; Delgado et al., 2008), ${ }^{7}$ como indicador síntesis de estos factores, ha sido un primer intento — si bien indirecto - para evaluar la relación entre las características específicas del espacio geográfico y la desigualdad en la población.

El ICUR es un índice desagregado a escala municipal que, además del nivel de urbanización, incluye en su composición dos elementos que reflejan indirectamente la consolidación y accesibilidad a empleos, bienes y servicios: i) la distancia real

$5 \longdiv { \text { La idea de observar cómo ciertos atributos se encarnan en el espacio geográfico y se asocian a la existencia de determina- } }$ dos tipos de empleo, ingreso y consumo se remite a, entre otros, Arriagada (2000), Bournazou (2008) y Ziccardi (2008). Ahí se discute la noción de 'bienes de consumo colectivo' propuesta en los años setenta por Castells (1977) y Lojkine (1976), donde el Estado provee los 'medios colectivos de consumo de mercancías', necesarios para la generación de ciertas relaciones sociales y la localización y reproducción de ciertos perfiles de fuerza de trabajo, que no son provistos por el capital. Estos medios colectivos incluyen la mayor parte de los tipos de infraestructura de comunicaciones; los servicios médicos, deportivos, culturales y educativos; y el transporte público. Esta propuesta ha sido recientemente retomada para explicar cómo la ausencia de los medios colectivos señalados tiene una influencia directa en la producción de desigualdad, tanto social como espacial. Fuera de su contexto original, la idea también ha servido para imaginar mecanismos destinados a disminuir la desigualdad, al distribuir de forma más concentrada algunos recursos clave (transporte, vías de comunicación, espacios de interacción) para acceder al empleo, al ingreso y al consumo.

6 En geografia, los trabajos que tratan en detalle el papel de las características materiales del espacio y su accesibilidad en la generación de desigualdad pertenecen, sobre todo, a la geografia de la salud (Curtis, 2004; Smyth, 2008; Meade \& Emch, 2010). También se ha recurrido a la noción de 'capital espacial' para expresar una idea similar acerca de la importancia del espacio geográfico. Sin embargo, estos autores utilizan el concepto de capital espacial de manera contradictoria; por ahora, la idea está sujeta a debate en tanto se define si es la unidad territorial o un sujeto quien encarna esta forma de capital (Centner, 2008; Marcus, 2008; Rérat \& Lees, 2011).

7 La selección de este índice está directamente relacionada con su viabilidad tanto de extensión como de resolución. Es un indicador síntesis de las características espaciales generales a escala nacional y para todos los municipios del país. 
por carretera de las cabeceras municipales al centro de la zona metropolitana más cercana (la que suele adoptar los valores más altos de consolidación); y ii) la alta calificación en el empleo, que incluye la infraestructura necesaria para soportar perfiles de ocupación profesionales y técnicos, funcionarios, personal directivo público y privado (Delgado et al., 2008, pp. 45-46). ${ }^{8}$ La ventaja de este índice respecto de otros tipos de información disponible sobre las características del territorio es, por un lado, su resolución a nivel municipal, que visibiliza procesos territoriales no observables a partir de información a escala de localidad o entidad federativa; y por otro, la disponibilidad de la información requerida para su construcción, con la posibilidad de actualizar el índice periódicamente a partir de información censal.

En la siguiente sección se abordan las características espaciales de México con relación a los fenómenos que sintetizan los tres índices seleccionados (IDH, Gini e ICUR), con el objetivo de presentar la distribución geográfica de la pobreza, desigualdad y grado de urbanización. Posteriormente, la asociación entre ellos será discutida y puesta a prueba con el objetivo de problematizar de manera más profunda sus componentes espaciales.

\section{Distribución del desarrollo humano, la desigualdad y el grado de urbanización en México}

Veremos ahora un perfil de la distribución geográfica del desarrollo humano, la desigualdad y la urbanización a escala municipal, así como las características socioeconómicas generales de grupos de municipios agregados en quintiles, de acuerdo con los valores de los índices. Los datos provienen del Conteo de Población y Vivienda de 2005, excepto los de ingreso, tomados del Censo de Población y Vivienda del año $2010 .^{9}$

La Figura 1 presenta la distribución nacional de los tres índices. El mapa del ICUR muestra dos grandes áreas de concentración urbana a nivel nacional y varios nodos urbanos en proceso de consolidación, casi todos en forma de corredor. La primera aglomeración abarca partes de las regiones centro y occidente, con extensiones hacia Chilpancingo, Tehuacán, Zacatecas y San Luis Potosí. La segunda aglomeración, de mucho menor extensión y poder económico, se forma alrededor

$8 \quad$ El ICUR es un índice cuya ventaja comparativa sobre la evaluación simple del nivel de urbanización (es decir, de la proporción de la población que vive en localidades mayores a 15.000 habitantes) es que integra seis variables desagregadas a escala municipal, las cuales están asociadas a la consolidación de la urbanización en el territorio. El índice está construido por medio del método de componentes principales y en conjunto explica el 80,8 por ciento de la varianza de la información. Las variables que integran el ICUR en su versión revisada son: 1) porcentaje de PEA no agrícola; 2) nivel de urbanización; 3) distancia real por carretera al centro metropolitano más cercano; 4) y 5) alta y baja calificación del empleo; y 6) inmigración. Los resultados del índice se estratificaron en cinco niveles por el método de estratificación de Jenk, de lo que resultan cinco grados de consolidación de la urbanización determinables para cada municipio: muy alto, alto, medio, bajo y muy bajo (Delgado et al., 2008, pp. 45-46).

9 La primera versión del artículo utilizaba datos de 2000 para calcular el ingreso y coeficiente Gini, ya que los datos de población más recientes eran los de 2005 (no incluyen el ingreso). A petición de un dictaminador anónimo se actualizaron los datos de ingreso, pero se mantuvo el resto de los datos para 2005. 
de Monterrey y se extiende hasta la Región Lagunera al oeste, además de una secuencia de ciudades fronterizas al norte (Ciudad Acuña, Piedras Negras, Nuevo Laredo, Reynosa y Matamoros). En cuanto a los nodos en consolidación, el principal de ellos está en el extremo de la península de Yucatán (desde Ciudad del Carmen hasta Cancún); el segundo, en el Istmo de Tehuantepec; el tercero, en el extenso corredor costero en Sonora-Sinaloa; y el más débil de todos, entre Chihuahua y Ciudad Juárez (Delgado \& Galindo, 2007).

Respecto del mapa del Índice de Desarrollo Humano, se observan también áreas de concentración que coinciden parcialmente con las aglomeraciones del ICUR. Sin embargo, el IDH presenta menos municipios con valores altos al sur de la aglomeración central y en los corredores del Istmo y Yucatán. A primera vista, los valores altos del IDH se asocian más con la concentración urbana que con la difusión periurbana del ICUR. En contraste, aparece un mayor número de municipios con valores altos de IDH al norte de la aglomeración central y a lo largo de la frontera con los Estados Unidos. Independientemente del sesgo que representa el mayor tamaño de los municipios en esta parte del país, una lectura superficial del IDH indicaría un mejor nivel de vida en el norte que en el resto del país, incluyendo el centro-occidente.

Por su parte, el coeficiente de Gini es consistente con los dos indicadores anteriores, sobre todo con el ICUR. En efecto, el tercer mapa muestra tres grandes áreas de concentración espacial con mayores niveles de desigualdad intermunicipal y que ocupan grandes intersticios mesorregionales entre áreas de concentración urbana. La primera abarca, básicamente, desde la sierra Tarahumara hasta la sierra de Durango, para disminuir drásticamente en la periferia —esta sí, urbana - de los núcleos urbanos de Chihuahua y La Laguna al este y de la llanura costera del Pacífico. La segunda aglomeración de desigualdad forma un arco entre las sierras Madre Occidental en Nayarit y la Oriental en el otro extremo geográfico, pasando por las sierras y llanuras áridas de Zacatecas y San Luis Potosí, que separa claramente las dos aglomeraciones principales (centro-occidente y Monterrey) definidas por el ICUR. Es pertinente notar que ninguna de estas tres áreas de mayor intensidad de la desigualdad cuenta con centros urbanos importantes, excepto la región sursureste, donde destacan Acapulco, Oaxaca y Tuxtla en medio de extensos bolsones de desigualdad.

Las características socioeconómicas básicas de los distintos municipios, de acuerdo con los valores de los distintos índices, se muestran en el Cuadro 1. La población está más concentrada en municipios con mayores niveles de desarrollo humano y/o urbanización, que en municipios con menores niveles de desigualdad. Mientras que 20 por ciento de los municipios con mayor nivel de desarrollo humano y consolidación urbana concentran a 67 y 71 por ciento de la población del país, respectivamente, en el otro extremo, 20 por ciento de los municipios con menor índice Gini (menor desigualdad) concentra a 25 por ciento de la población. 


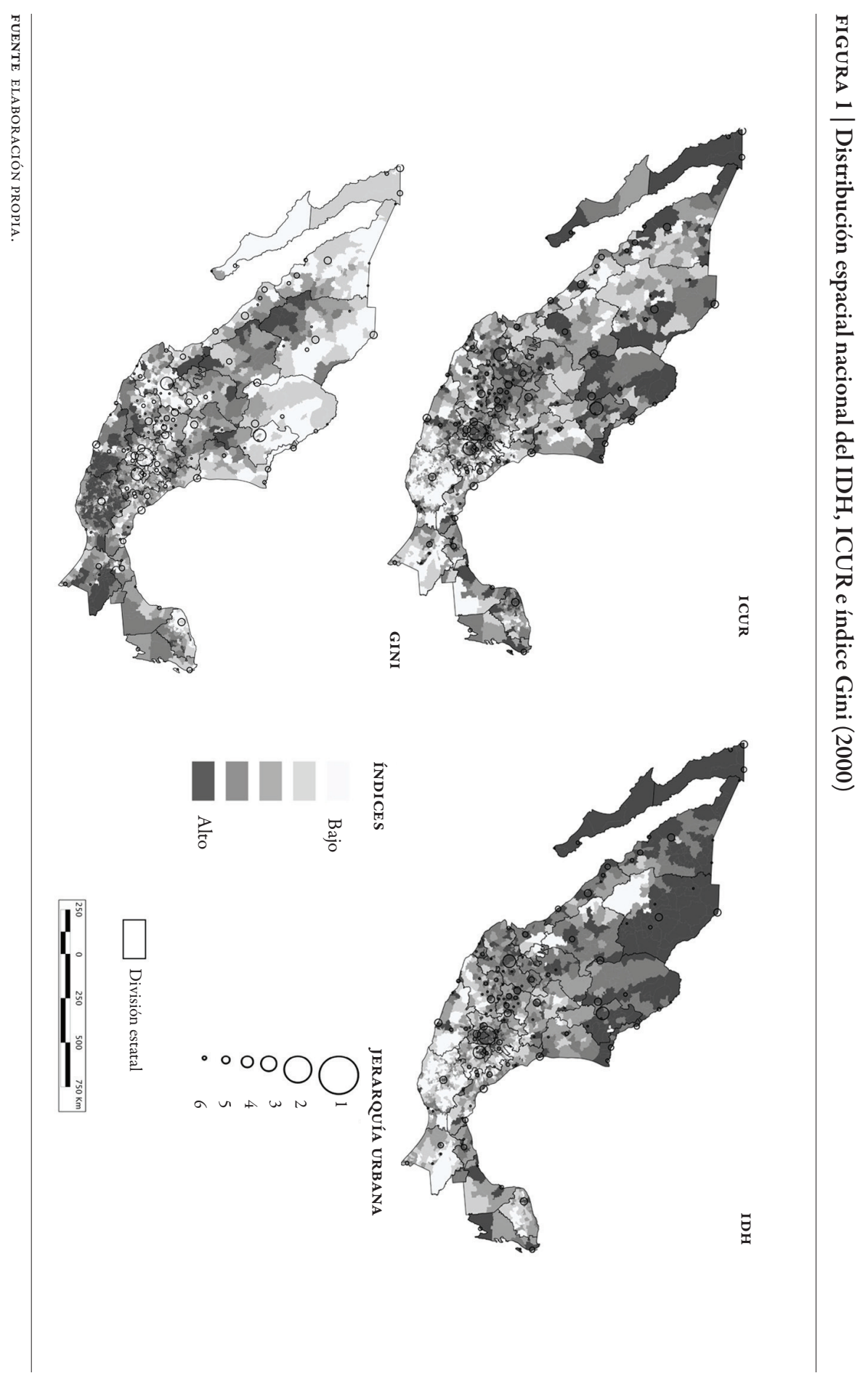


CUADRO 1 | Características seleccionadas para municipios de México por quintiles de IDH, ICUR y Gini

(a) Quintiles municipales, índice de desarrollo humano (IDH)

\begin{tabular}{lrrrrrr}
\hline & IER & 2DO & 3RO & 4TO & 5TO & NACIONAL \\
\hline Población (millones) & 5,1 & 6,6 & 9,9 & 15,0 & 66,9 & 103,5 \\
\% pob. nacional & 4,9 & 6,4 & 9,6 & 14,5 & 64,6 & 100 \\
\% derechohabientes & 15,9 & 23,4 & 30,9 & 39,7 & 55,4 & 46,8 \\
\% viviendas con agua & 56 & 67,7 & 74,4 & 84,4 & 90,4 & 85,4 \\
\% viviendas con drenaje & 38,3 & 57,9 & 70 & 83,4 & 91,6 & 84,4 \\
\% viviendas con piso de tierra & 54,9 & 31,3 & 19,8 & 11,5 & 3,6 & 9,9 \\
\% población urbana (>2500) & 19,4 & 32,3 & 44 & 63,1 & 92,7 & 76,3 \\
Escolaridad promedio (años) & 4,3 & 5,4 & 6,1 & 7 & 9,2 & 8,1 \\
Ocupantes por vivienda promedio & 5,1 & 4,6 & 4,5 & 4,3 & 4 & 4,6 \\
Ingreso por trabajo per cápita en hogares (mediana) & 194 & 642 & 942 & 1.285 & 2.143 & 704 \\
\hline
\end{tabular}

(b) Quintiles municipales, índice de consolidación urbano regional (ICUR)

\begin{tabular}{lcccccc}
\hline & IER & 2DO & 3RO & 4TO & 5TO & NACiONAL \\
\hline Población (millones) & 4,7 & 6,3 & 8,0 & 12,8 & 71,8 & 103,5 \\
\% pob. nacional & 4,5 & 6 & 7,7 & 12,4 & 69,3 & 100 \\
\% derechohabientes & 19,1 & 26,5 & 34,1 & 37,7 & 53,4 & 46,8 \\
\% viviendas con agua & 60,4 & 67,7 & 75,1 & 81,4 & 90 & 85,4 \\
\% viviendas con drenaje & 47,3 & 60,8 & 70,7 & 77,7 & 90,9 & 84,4 \\
\% viviendas con piso de tierra & 47,4 & 29,8 & 20,6 & 14,8 & 4,2 & 9,9 \\
\% población urbana (>2500) & 15,1 & 28 & 41,8 & 56,4 & 91,9 & 76,3 \\
Escolaridad promedio (años) & 4,7 & 5,5 & 6,1 & 6,7 & 9 & 8,1 \\
Ocupantes por vivienda promedio & 4,8 & 4,5 & 4,3 & 4,4 & 4,1 & 4,6 \\
Ingreso por trabajo per cápita en hogares (mediana) & 257 & 686 & 952 & 1.171 & 2.142 & 1.714 \\
\hline
\end{tabular}

(c) Quintiles municipales, índice de Gini

\begin{tabular}{lrrrrrr}
\hline & I ER & 2DO & 3RO & 4TO & 5 TO & NACIONAL \\
\hline Población (millones) & 24,7 & 42,7 & 23,0 & 8,3 & 4,7 & 103,5 \\
\% pob. nacional & 23,9 & 41,2 & 22,2 & 7,0 & 6,6 & 100 \\
\% derechohabientes & 49,0 & 52,7 & 46,2 & 29,28 & 14,97 & 46,8 \\
\% viviendas con agua & 87,9 & 88,7 & 85,2 & 69,3 & 56,6 & 85,4 \\
\% viviendas con drenaje & 88,0 & 88,8 & 84,2 & 66,1 & 41,5 & 84,4 \\
\% viviendas con piso de tierra & 6,4 & 5,3 & 9,4 & 26,8 & 49,9 & 9,9 \\
\% población urbana (>2500) & 86,8 & 84,4 & 73,9 & 50,3 & 39 & 76,3 \\
Escolaridad promedio (años) & 8,1 & 8,7 & 8,3 & 6,1 & 4,6 & 8,1 \\
Ocupantes por vivienda promedio & 4,2 & 4,1 & 4,1 & 4,4 & 4,9 & 4,6 \\
Ingreso por trabajo per cápita en hogares (mediana) & 1.809 & 2.000 & 1.714 & 750 & 18 & 1.714 \\
\hline
\end{tabular}

FUente CÁlCulos de los autores con base en datos del Instituto Nacional de Estadística y Geografía (INEGI), MÉXICO, 2005; 2010. 
Las distintas variables se comportan según lo esperado. Los grupos de municipios con mayor IDH tienen, por un lado, mayores porcentajes de derechohabientes a servicios de salud, viviendas con servicios de agua y drenaje, población en localidades urbanas, así como mayores niveles de escolaridad e ingreso. Por el otro lado, tienen un menor porcentaje de viviendas con piso de tierra y un menor número de ocupantes por vivienda. El mismo patrón se observa para todos los grupos de municipios en el caso del ICUR.

En el caso del Gini, el patrón es inverso (por la naturaleza de los valores del índice), aunque el cambio entre quintiles es mucho menos marcado. De hecho, son pocas las diferencias en los valores de los indicadores entre los municipios del primer y segundo quintiles y, en varios casos, no siguen la correlación negativa que se observa entre el resto de los quintiles. La diferencia entre municipios se vuelve un poco más marcada entre el segundo y el tercer quintiles y el comportamiento de las variables sugiere que mayores niveles de desigualdad están asociados con menores niveles de escolaridad, ingreso, urbanización y disponibilidad de servicios. En general, se podría intuir una distribución más homogénea de la población y sus estratos entre municipios con distintos valores de Gini que en el caso del IDH y el ICUR; o quizá, una menor variación de la desigualdad en términos territoriales que la del desarrollo humano, y por supuesto que de la consolidación urbana.

Ahora bien, a partir de esta distribución general, es necesario investigar la asociación espacial entre los diferentes contextos sociales reflejados en los tres índices, para evidenciar el papel de las características espaciales en el desarrollo humano y la desigualdad. A continuación se presenta en detalle la metodología empleada para el análisis espacial de los indicadores, a través de la autocorrelación espacial entre ellos, misma que se discutirá en la parte final del artículo.

\section{Metodología}

A partir de los índices anteriores, se generó una metodología propia para realizar un análisis espacial que permitiera medir: i) la asociación global de los índices en el espacio (autocorrelación espacial); ii) la similitud entre municipios adyacentes, con el fin de identificar regiones de altos y bajos niveles de pobreza, desigualdad y urbanización mediante su tipificación (clusters espaciales); y iii) la influencia de los niveles de urbanización regional y el acceso a los centros urbanos como determinantes de desigualdad.

\section{Autocorrelación espacial}

Para medir si los valores de los índices se aglomeran en el espacio, se calculó primero el grado de autocorrelación espacial global para cada índice utilizando la I de Moran. La I de Moran varía de - 1 a 1, donde - 1 significa autocorrelación espacial negativa perfecta, 0 significa ausencia de correlación y 1 significa autocorrelación espacial positiva perfecta (Figura 2). De resultar positivos dichos valores, ello indicaría que los municipios con altos niveles de pobreza, desigualdad o baja urbanización, respectivamente, se aglomeran espacialmente; y que aquellos municipios con valores bajos de los mismos índices, también se aglomeran espacialmente. La I de Moran está dada por: 


$$
I=\frac{N}{\sum_{i} \sum_{j} w_{i j}} \cdot \frac{\sum_{i} \sum_{j} w_{i j}\left(x_{i}-\bar{x}\right)\left(x_{j}-\bar{x}\right)}{\sum_{j}\left(x_{i}-\bar{x}\right)^{2}}
$$

(Ecuación 1)

donde:

$x_{i}=$ Valor del índice para el caso $i$

$\bar{x}=$ Media global del índice

$x_{j}=$ Valores de los vecinos

$w_{i j}$ es una matriz de ponderación por distancia entre los valores $i$ y $j$

$N=$ Número de casos

FIGURA 2 | Representación de valores extremos de la I de Moran

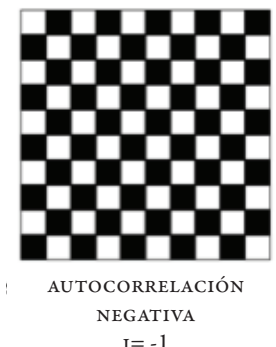

$\mathrm{I}=-1$

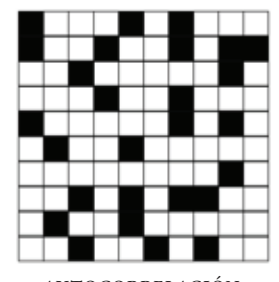

AUTOCORRELACIÓN

NULA

$\mathrm{I}=0$

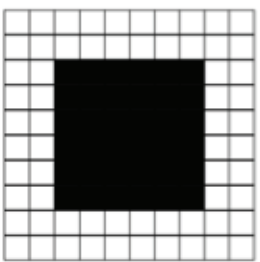

AUTOCORRELACIÓN

POSITIVA

FUENTE ELABORACIÓN PROPIA.

\section{Autocorrelación local}

El índice de autocorrelación espacial anterior permite conocer el grado de aglomeración de una variable, pero no indica dónde sucede dicha aglomeración o si ella se presenta, a diferentes niveles, en distintas regiones. Esta cualidad puede obtenerse mediante la I de Moran local, un estadístico de autocorrelación espacial local que mide la proporción de la variación respecto de la variación media, ponderada por la variación con la distancia (Anselin, 1995). La I de Moran local permite identificar conglomerados espaciales debido a una probabilidad de pertenecer o no al promedio de los vecinos cercanos, de acuerdo con criterios de significación estadística. La I de Moran local está dada por:

$$
I_{i}=\frac{\left(x_{i}-\bar{x}\right)}{\sum_{i=1}^{n}\left(x_{i}-\bar{x}\right)^{2} / n} \sum_{j=1}^{n} w_{i j}\left(x_{j}-\bar{x}\right)
$$

(Ecuación 2)

donde, los valores son los mismos que los de la Ecuación 1. 
Mediante este estadístico, se identificaron cuatro tipos de conglomerados (Cuadro 2), además de una categoría de no asociación local donde la variación entre vecinos es el rango promedio del área de estudio.

CUADRO 2 | Tipos de conglomerados identificables mediante el uso la I de Moran local.

\begin{tabular}{ll}
\hline TIPO DE CLUSTER & INTERPRETACiÓN \\
\hline HH (Alto-alto) & Valores altos entre vecinos con valores altos \\
LL (Bajo-bajo) & Valores bajos entre vecinos con valores bajos \\
HL (Alto-bajo) & Valores altos entre vecinos con valores bajos (casos extremos) \\
LH (Bajo-alto) & Valores bajos entre vecinos con valores altos (casos extremos) \\
\hline
\end{tabular}

FUENTE ELABORACIÓN PROPIA.

Una vez identificada la pertenencia de cada municipio a distintos tipos de conglomerados espaciales, se analizó la relación entre ellos y el valor medio de cada índice. Adicionalmente, se midió la asociación entre grupos de municipios identificados en cada índice con los de otros índices, mediante la construcción de tablas de contingencia y el cálculo del estadístico $\lambda$ (lambda). Dicho procedimiento permitió comenzar a identificar la asociación espacial entre los diferentes índices y observar las formas de aglomeración local.

\section{Desigualdad, pobreza y urbanización}

La medición de la auto-asociación espacial de los índices utilizados y su representación cartográfica permitieron, de manera descriptiva, intuir una asociación espacial entre ellos. Con el propósito de confirmar esta asociación y medirla cuantitativamente, se corrieron dos modelos de regresión.

Primeramente, se corrió una regresión lineal de mínimos cuadrados ordinarios (RMCO), que predice el grado de desigualdad en la distribución del ingreso (medido en términos del coeficiente Gini municipal), en función del grado de urbanización (medido en términos del ICUR) y utilizando el IDH como variable de control. Posteriormente, esa misma regresión se corrió, pero modelada como regresión geográficamente ponderada (RGP). A diferencia de una RMCO, la RGP toma en cuenta (además de los valores municipales de los regresores de cada caso), los valores de los municipios cercanos y los pondera mediante la distancia. En nuestro caso, utilizamos una ponderación bicúbica. El procedimiento permite que los coeficientes varíen espacialmente, minimizando los residuos cuando, en efecto, existe una asociación espacial.

Teniendo ambos modelos de regresión la proporción de la variación explicada ( $\mathrm{R}^{2}$ ajustada) de la RMC, se restó de la $\mathrm{R}^{2}$ ajustada de la RGP, dando como resultado la proporción de la variación de la desigualdad que explica la distribución espacial. La fórmula general de una RGP está dada por: 
$\hat{y}=\beta_{0}\left(u_{i}, v_{i}\right)+\sum_{k} \beta_{k}\left(u_{i}, v_{i}\right) x_{i k}$

(Ecuación 3)

donde:

$\beta_{0}\left(u_{i}, v_{i}\right)=$ Constante para cada observación fijada en las coordenadas $\left(u_{i}, v_{i}\right)$

$\beta_{k}\left(u_{i}, v_{i}\right)=$ Los coeficientes de las variables independientes, para cada observación con ponderación $u_{i} y v_{i}$.

$x_{i k}=$ Valor de la variable independiente en el sitio $i$

y la ponderación bicúbica está dada por:

$\left.w\{u, v\}(g)=\left(1-d\{u, v\}^{2}\right) / d^{2}\right)^{2}$

(Ecuación 4)

si $d_{i j} \leq d$, de lo contrario, $w\{u, v\}(\mathrm{g})=0$

donde:

$w\{u, v\}(\mathrm{g})=$ a la matriz de ponderaciones espaciales entre observaciones

$d=$ la distancia donde la ponderación se convierte en 0 , calculada mediante una optimización de ancho de banda utilizando el criterio de información de Akaike (Bivand \& Yu, 2008)

$d\{u, v\}$ es la distancia entre observaciones

\section{Limitaciones de la metodología empleada}

Antes de presentar los resultados, vale la pena señalar algunas limitaciones de la metodología utilizada. La primera es la elección de índices. El coeficiente Gini como medición de desigualdad puede ser cuestionado, principalmente porque no permite saber la proporción del ingreso que se encuentra en manos de un determinado sector social. En segundo lugar porque, aunque el Gini permite conocer la desigualdad en su distribución, la medición de la riqueza y el bienestar no se basa únicamente en el ingreso. Además, es cuestionable que la igualdad en la distribución del ingreso sea necesariamente un indicador positivo, ya que en el caso de un municipio muy pobre, una perfecta igualdad en la distribución del ingreso indica que todos sus miembros serían igualmente pobres. En tercer lugar, la agregación a nivel municipal es cuestionable: por un lado supone que cada municipio es una isla en la que el ingreso debiera estar igualmente distribuido, sin tomar en cuenta el ingreso que se genera en sus alrededores o incluso en el país mismo; por otro lado, aun cuando la agregación municipal no permite conocer las desigualdades espaciales al interior de los municipios, una menor agregación espacial agudizaría el problema de la isla de distribución. 
Otra limitación es que la ponderación con distancias euclidianas supone una red de transporte intermunicipal perfecta, homogénea y en una planicie. Esto representa un problema, pues los lugares más pobres suelen localizarse en zonas con relieves más abruptos y dotadas con infraestructura de transporte más débil, por lo que es posible que el modelo de regresión subestime la relación espacial entre la desigualdad y la pobreza, pero sobre todo, la accesibilidad a centros urbanos. Finalmente, cabe señalar la necesidad de incluir información política y cultural en la elección de variables independientes, la misma que no siempre es fácil de traducir a términos cuantitativos, ni se encuentra sistematizada para todo el país.

En investigaciones futuras, habrá que investigar metodologías que permitan establecer ventanas móviles regionales para calcular la desigualdad de cada lugar, en términos de lo que sucede en un radio de distancia (ancho de banda) en torno suyo. Asimismo, habrá que experimentar el uso de tiempos de traslado, tomando en cuenta los tipos de infraestructura carretera, así como el relieve. Ello requerirá de un fuerte poder de cómputo, que permita estimar anchos de banda para regresiones espaciales con cientos de miles de casos (localidades), para posteriormente calcular tiempos de traslado desde cada una de las localidades del país a aquellas localidades que se encuentren conectadas por la red vial, a un tiempo de traslado menor o igual al ancho de banda seleccionado.

\section{Resultados}

\section{Autocorrelación espacial global}

Los resultados de la I de Moran Global muestran una autocorrelación espacial moderada de los índices. Esto es un resultado esperado, ya que es lógico que, aunque los índices varíen en el espacio, la aglomeración de los valores más altos se presente en distintas localizaciones al mismo tiempo (Cuadro 3). En el caso del ICUR, por ejemplo, el índice aumentará necesariamente en la cercanía de las ciudades. Debido a que las ciudades se encuentran físicamente separadas una de otra, es de esperarse que la variación de la asociación se presente a lo largo del territorio nacional. Ese patrón es más claro si se revisan los mapas de la sección "Distribución del desarrollo humano, la desigualdad y el grado de urbanización en México". En el caso del coeficiente Gini y el IDH, sucede algo similar. Los municipios con los valores más altos son, por lo general, adyacentes entre sí, al igual que aquellos municipios con los valores más bajos.

CUADRO 3 | Autocorrelación espacial de tres índices

\begin{tabular}{ll}
\hline ÍNDICE & VALOR DE I DE MORAN \\
\hline Gini & 0,64 \\
IDH & 0,40 \\
ICUR & 0,61 \\
\hline
\end{tabular}

FUENTE CÁLCULOS DE LOS AUTORES. 


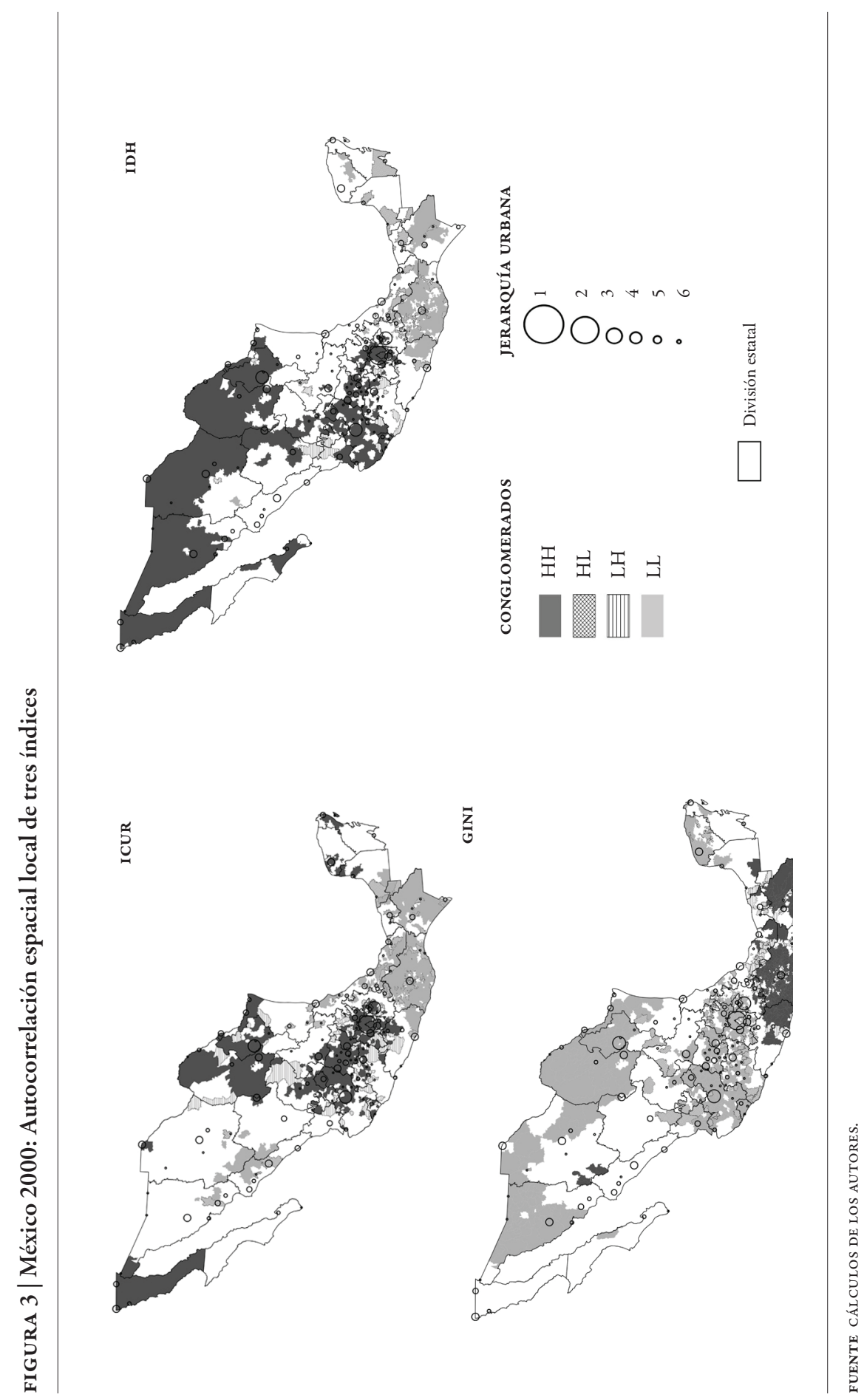




\section{Autocorrelación espacial local}

Considerando la posibilidad de encontrar distintos lugares con valores autocorrelacionados a lo largo del territorio, la posibilidad de hallar patrones espaciales resulta más factible utilizando la I de Moran local. La Figura 3 muestra el resultado de los conglomerados producidos mediante este procedimiento para los tres índices; y el Cuadro 4, las tablas de contingencia y el estadístico de asociación.

Debido a que $\lambda$ es un estadístico de reducción proporcional de error, sus valores hacen evidente que existe una asociación alta en la aglomeración de los índices. Es decir, los municipios que se aglomeran por tener un menor grado de desigualdad son, por lo general, municipios que se aglomeran por tener mayores niveles de urbanización y de desarrollo humano. De igual forma y en el otro extremo, las aglomeraciones de municipios con mayor desigualdad tienden a coincidir con aglomeraciones de municipios con bajos niveles de urbanización y menor desarrollo humano.

CUADRO 4 |Tablas de contingencia y asociación $(\lambda)$ entre Gini, IDH e ICUR

\begin{tabular}{|c|c|c|c|c|c|c|c|c|c|c|c|c|c|c|c|}
\hline \multicolumn{6}{|c|}{ IDH } & \multicolumn{5}{|c|}{ ICUR } & \multicolumn{5}{|c|}{ ICUR } \\
\hline GINI & $\mathrm{HH}$ & HL & LH & LI & & GINI & $\mathrm{HH}$ & IL & & & IDH & $\mathrm{HH}$ & HL & & $\mathrm{LL}$ \\
\hline $\mathrm{HH}$ & 0 & 14 & & 0 & 517 & $\mathrm{HH}$ & 2 & 33 & 3 & 580 & $\mathrm{HH}$ & 473 & 2 & 11 & 1 \\
\hline HL & 3 & ( & 0 & 14 & 15 & HL & 6 & 0 & 47 & 13 & $\mathrm{HL}$ & 1 & 129 & 0 & 19 \\
\hline LH & 0 & 13 & & 0 & 30 & LH & 1 & 139 & 0 & 48 & LH & 4 & 0 & 12 & 0 \\
\hline LL & 576 & & 1 & 7 & 4 & LL & 564 & 3 & 31 & 3 & LL & 2 & 42 & 0 & 519 \\
\hline
\end{tabular}

FUENTE CÁLCULOS DE LOS AUtORES.

Ahora bien, aunque hay un alto número de municipios que se aglomeran en algún tipo de conglomerado, más de la mitad tiene valores que generan una variación aleatoria que no permite su aglomeración. Sin embargo, es evidente que en estos municipios también existen valores de desigualdad, urbanización y desarrollo humano. Falta entonces conocer el efecto conjunto del IDH e ICUR sobre el Gini, tomando en cuenta no solo la variación de los índices dentro de los municipios, sino también los valores en el contexto regional.

\section{Desigualdad, pobreza y urbanización}

Los resultados de la RGP se muestran en el Cuadro 5 junto con los estadísticos de diagnóstico que la comparan con una RMCO. La RGP, con una $\mathrm{R}^{2}$ ajustada de 0,66 , explica más de tres veces mejor la variación del Gini municipal que el modelo de regresión simple $\left(\mathrm{R}^{2}{ }_{\mathrm{aj}}=0,21\right)$. De igual forma, el resto de los parámetros muestra que la RGP es mucho más robusta que la RMCO: tiene una suma de residuos cuadrados menor y un valor menor del criterio de Akaike. El hecho de que la 
$\mathrm{R}^{2}$ ajustada entre la RMCO y la RGP se triplique, se debe a la asociación espacial (regional) que existe entre los índices. Ciertamente, la interpretación directa de los coeficientes resulta poco intuitiva, ya que los valores intermedios de los índices no tienen una interpretación directa. No obstante, de acuerdo con los coeficientes beta, la urbanización pesa casi dos veces más que el desarrollo humano sobre la variación en la distribución del ingreso.

En la RGP, la variación del Gini tanto en función del ICUR como en función del IDH es la esperada para todos los cuartiles de casos municipales. Es decir que, a medida que la consolidación urbana aumenta regionalmente, en términos del índice Gini, la desigualdad tiende a disminuir. Lo mismo sucede con el desarrollo humano que, a medida que aumenta, disminuye la desigualdad. Aunque para ambas variables el valor máximo es positivo, solo en unos cuantos municipios (que agrupan cerca del 4 por ciento de la población) resultó positiva la asociación entre la urbanización y la desigualdad o entre el desarrollo humano y la desigualdad. Al realizar una inspección de esos casos, no se encontró ninguna característica peculiar, excepto de que se trataba, en la mayoría de ellos, de municipios o costeros o fronterizos. Debido a que esos municipios no están rodeados por completo de otros y, por lo tanto, tienen un número menor de regresores que el promedio, se puede suponer simplemente que son casos atípicos.

CUADRO 5 | Regresión geográficamente ponderada ${ }^{a}$ del coeficiente Gini municipal, 2000

\begin{tabular}{|c|c|c|c|c|c|c|c|}
\hline & MIN. & IER CUARTIL & MEDIANA & 3ER CUARTIL & MAX. & GLOBAL & BETA GLOBAL \\
\hline Constante & $-2,09$ & 0,63 & 0,73 & 0,87 & 5,2 & 0,81 & \\
\hline ICUR & $-0,16$ & $-0,09$ & $-0,05$ & $-0,03$ & 0,35 & $-0,06$ & $-0,46$ \\
\hline IDH & $-6,12$ & $-0,41$ & $-0,25$ & $-0,15$ & 3,28 & $-0,36$ & $-0,28$ \\
\hline \multicolumn{2}{|c|}{ DIAGNóstico } & \multicolumn{3}{|c|}{ PARÁMETROS GLOBALES (RMCO) } & PARÁMETR & RGP & \\
\hline \multicolumn{2}{|c|}{ Suma de residuos ${ }^{\wedge}$} & \multicolumn{3}{|c|}{23,4} & \multicolumn{3}{|c|}{13,2} \\
\hline \multicolumn{2}{|l|}{$\mathrm{R}^{2}$ ajustada } & \multicolumn{3}{|c|}{0,21} & \multicolumn{3}{|c|}{0,66} \\
\hline \multicolumn{2}{|c|}{ Regresores efectivos } & \multicolumn{3}{|c|}{2} & \multicolumn{3}{|c|}{183,2} \\
\hline \multicolumn{2}{|c|}{ Criterio de Akaike } & \multicolumn{3}{|c|}{-4.515} & \multicolumn{3}{|c|}{$-5.749,2$} \\
\hline
\end{tabular}

FUente CÁlculos de los autores.

${ }^{A}$ FUNCIÓN GAUSIANA DE PONDERACIÓN ESPACIAL CON ANCHO DE BANDA OPTIMIZADA A 61,2 KM, MEDIANTE DROP -1 CROSSVALIDATION (BIVAND \& DANLIN, 2008; FOTHERINGHAM, BRUNSDON \& CHARLTON, 2000).

\section{Conclusiones}

El propósito de este artículo ha sido medir la asociación entre la desigualdad en la distribución del ingreso, con el desarrollo social y la distribución geográfica de la urbanización en México, a objeto de determinar los factores espaciales que inciden en la generación de la desigualdad. Para ello utilizamos las medidas de autocorrela- 
ción espacial global y local entre los Índices de Desarrollo Humano, la desigualdad del ingreso medida en términos del Índice de Gini por municipio, y el Índice de Consolidación Urbano-Regional (ICUR). A partir de estas medidas se determinó la relación que guarda, en términos espaciales, el Gini con los otros dos índices, mediante una regresión geográficamente ponderada. Se encontró que los tres índices tienen una autocorrelación espacial global moderada. Asimismo, que con criterios de autocorrelación local es posible encontrar aglomeraciones espaciales de municipios con valores similares de los tres índices. Más aún, que hay una asociación no paramétrica entre los distintos tipos de conglomerados que el procedimiento de autocorrelación local genera para cada índice. Los municipios con valores bajos de Gini (menor desigualdad) suelen ser municipios con valores altos de desarrollo humano y valores altos de urbanización.

El hallazgo más importante es que, debido a que existe una mayor asociación entre el Gini municipal y el ICUR que entre el Gini y el IDH, los factores geográficos que influyen en el acceso a bienes y servicios tendrían una mayor influencia en la desigualdad que los niveles de desarrollo humano. Las características del espacio geográfico desempeñan, así, un papel clave al explicar la variación de la desigualdad en el territorio nacional, en función del desarrollo humano y la urbanización.

Mientras que el modelo de regresión lineal aquí propuesto toma en cuenta los valores municipales de ICUR e IDH para predecir el Gini territorial municipal, el modelo geográficamente ponderado valora, además, las variables independientes de municipios cercanos, ponderando los valores por la distancia. Los resultados de este último modelo explican el doble de la variación en el Gini, respecto de aquellos del modelo de regresión lineal. En términos generales, se encontró que a medida que aumentan los niveles de urbanización y de desarrollo humano, el Gini tiende a disminuir.

De nueva cuenta, el papel del espacio geográfico es determinante porque, incluso si un municipio no tiene un alto nivel de urbanización e IDH, cuando se encuentra cerca de otro(s) municipio(s) con valores altos de urbanización y desarrollo humano, también tenderá a tener un menor grado de desigualdad. Así, para el caso del desarrollo humano, si un municipio pobre está rodeado de municipios en la misma circunstancia, tenderá a ser más desigual que otro municipio pobre cuyos vecinos cercanos tienen un mayor nivel de desarrollo humano. En nuestra opinión, esto significa que, en igualdad de circunstancias, el patrón espacial menos óptimo para una población es aquel donde la pobreza está rodeada de pobreza.

Para el caso de la urbanización, la explicación puede ser similar. Aunque un municipio no cuente con bienes y servicios consolidados, el estar cerca de otro que sí los posee le representa ventajas clave de accesibilidad, que influyen sobre la distribución de la riqueza. En nuestra opinión, estos resultados del modelo sugieren la necesidad de un nivel de planeación regional en torno a centros de distintas jerarquías que puedan proveer de servicios urbanos a sus áreas periurbanas y regionales, y no únicamente bajo la óptica de planeación municipal o metropolitana, en el mejor de los casos. Los municipios "cercanos" deben ser considerados en la estimación de la demanda de servicios, no solamente local sino regional. 
Los hallazgos de este trabajo fortalecen la hipótesis de que la concentración de bienes y servicios clave en el espacio (reflejadas por el ICUR) explica buena parte de la desigualdad interna de los municipios y representa una dimensión regional importante de atender en la planeación de políticas ad hoc para disminuir la desigualdad. Es posible que el ICUR prediga mejor la desigualdad, porque los factores territoriales asociados a la accesibilidad a bienes, servicios e infraestructuras generan mayores oportunidades de empleo, ingreso y consumo. La existencia de estos "activos" territoriales puede influir en la generación de condiciones de desarrollo humano reflejadas en el IDH y en la disminución de las disparidades sociales en el acceso a los recursos que permiten el ingreso, condiciones que incluyen no solo bajas distancias y una determinada densidad de bienes y servicios, sino también la existencia extendida de actividades económicas de mayor calificación. OEURE

\section{Referencias bibliográficas}

Alasia, A. (2002). Alternative measures of territorial disparity: an application to Canada. Ponencia presentada en la reunión Working Party on Territorial Indicators. Territorial Development Services, Organisation for Economic Co-operation and Development (OECD), París.

Anselin, L. (1995). Local indicators of spatial association. Geographical Analysis, 27(2), 93-115. En http://www.dces.wisc.edu/documents/articles/curtis/cesoc977/Anselin1995.pdf

Arriagada, C. (2000). Pobreza en América Latina: Nuevos escenarios y desafíos de politicas para el hábitat urbano. Serie Medio Ambiente y Desarrollo, Cuaderno de trabajo 27. Santiago: Comisión Económica para América Latina y el Caribe (Cepal).

Atkinson, A. (1970). On measurement of inequality. Journal of Economic Theory, 2(3), 244-263.

Baharoglu, D. \& Kessides, C. (2001). Urban poverty. En J. Klugman (Ed.), A Sourcebook for Poverty Reduction Strategies (pp. 124-159). Washington: World Bank. En http://siteresources. worldbank.org/INTPRS1/Resources/383606-1205334112622/4418_chap16.pdf

Bivand, R. \& Yu, D. (2008). Spgwr: Geographically weighted regression. R package version 0.5-4. http://ncg.nuim.ie/ncg/GWR/index.htm

Boltvinik, J. (2004). Métodos de medición de la pobreza. Una tipología. Limitaciones de los métodos tradicionales y problemas de los combinados. En J. Boltvinik \& A. Damián (Coords.), La pobreza en México y el mundo. Realidades y desafíos (pp. 437-475). México: Siglo XXI Editores/Gobierno de Tamaulipas.

Bournazou, E. (2008). La segregación social del espacio y la dimensión territorial en los estudios de pobreza urbana. En R. Cordera, P. Ramírez \& A. Ziccardi (Coords), Pobreza, desigualdad y exclusión social en la ciudad del Siglo XXI (pp. 394-401). México: Instituto de Investigaciones Sociales (IIS), Universidad Autónoma de México (UNAM)/Siglo XXI Editores,

Brunsdon, C., Stewart-Fotheringham, A. \& Charlton, M. E. (1996). Geographically weighted regression: A method for exploring spatial nonstationarity. Geographical Analysis, 28(4), 281298. doi: 10.1111/j.1538-4632.1996.tb00936.x 
Centner, R. (2008). Places of privileged consumption practices: Spatial capital, the dot-com habitus and San Francisco's Internet boom. City and Community, 7(3), 193-223. doi: 10.1111/j.1540-6040.2008.00258.x

Castells, M. (1977). The urban question. Londres: Edward Arnold.

Consejo Nacional de Evaluación de la Política de Desarrollo Social (Coneval), México (2007). Los mapas de pobreza en México. Anexo Técnico-metodológico. México, D.F.: Autor. En http:// medusa.coneval.gob.mx/cmsconeval/rw/resource/coneval/med_pobreza/1209.pdf

Consejo Nacional de Población (Conapo), México (2006). Índices de marginación 2005. México, D.F.: Autor. En www.conapo.gob.mx/publicaciones/margina2005/IM2005_principal.pdf

Cortés, F. (2002). Consideraciones sobre la marginalidad, marginación, pobreza y desigualdad en la distribución del ingreso. Papeles de Población, 31 (enero-marzo), 9-24. En http://redalyc. uaemex.mx/pdf/112/11203101.pdf

Curtis, S. (2004). Health and inequality. Geographical perspectives. Londres: Sage.

Delgado, J. \& Galindo, C. (2007). Índice de Consolidación Urbano-Regional 2000. En A. CollHurtado (Coord.), Nuevo atlas nacional de México (Cap. Sociedad. Tema: Aglomeraciones urbanas multiescala, S XIII-1). México: Instituto de Geografía, UNAM.

Delgado, J., Galindo, C. \& Ricárdez, M. (2008). La difusión de la urbanización o cómo superar la dicotomía rural-urbana. En J. Delgado (Coord.), La urbanización difusa de la Ciudad de México. Otras miradas sobre un espacio antiguo (pp. 43-74). México: Instituto de Geografía, Universidad Nacional Autónoma de México (UNAM).

Delgado, J., Larralde, A. \& Anzaldo, C. (1999). La corona regional de la Ciudad de México. Primer anillo exterior en formación. En J. Delgado \& B. Ramírez (Coords.), Transiciones. La nueva formación territorial de la Ciudad de México (pp.171-194). México: Universidad Autónoma Metropolitana/Plaza y Valdés.

Economic Commission for Latin America and the Caribbean (ECLAC). (2010). Time for equality. Closing gaps, opening trails. Brasilia: Autor.

Escobal, J. \& Torero, M. (2005). Adverse geography and differences in welfare in Peru. En R. Kanbur \& A. Venables, Spatial inequality and development (pp. 77-122). Helsinki: UNU-Wider.

Fotheringham, A. S., Brunsdon, C. \& Charlton, M. E. (2000). Quantitative Geography. Londres: Sage.

Galindo, C. (2007). Urbanización difusa y reconfiguración rural-urbana en el Centro de México. Tesis de Maestría en Geografía, Universidad Nacional Autónoma de México (UNAM).

Getis, A., Mur, J. \& H. Zoller (2004). Spatial econometrics and spatial statistics. Basingstoke: Palgrave Macmillan.

Goerlich, F. \& Villar, A. (2009). Desigualdad y bienestar social. De la teoría a la práctica. Madrid: Fundación BBVA.

Grusky, D. \& Kanbur, R. (2006). Introduction. The conceptual foundations of poverty and inequality measurement. En D. Grusky \& R. Kanbur, Poverty and inequality (pp. 1-29). Stanford: Stanford University Press.

Kanbur, R. \& Venables, A. (2005). Spatial inequality and development. En R. Kanbur \& A. Venables (Eds.), Spatial inequality and development (pp. 2-11). Oxford: Oxford University Press.

Kanbur, R. (2005). Growth, inequality and poverty: Some hard questions. Journal of International Affairs, 58(2), 223-232. En http://www.arts.cornell.edu/poverty/kanbur/GroIneqPov. pdf 
Lojkine, J. (1976). Contribution to a Marxist theory of urbanization. En C. Pickvance (Ed.), Urban sociology: Critical essays (pp. 119-146). Londres: Tavistock.

Marcus, L. (2008). Spatial capital and how to measure it. En T. Haas (Ed.), New urbanism and beyond (pp. 135-139). Nueva York: Rizzoli.

Meade, M. \& Emch, M. (2010). Medical Geography [3ª ed.]. Nueva York: Guilford Press.

Moran, P. A. P. (1950). Notes on continuous stochastic phenomena. Biometrika, 37(1-2), 17-23.

Quah, D. (1996). Regional convergence clusters across Europe. European Economic Review, 40, 951958. En http://dx.doi.org/10.1016/0014-2921(95)00105-0

Rérat, P. \& Lees, L. (2011). Spatial capital, gentrification and mobility: Evidence from Swiss core cities. Transactions of the Institute of British Geographers, 36(1), 126-142. Publicado online por primera vez en sept. 28, 2012, doi: 10.1111/j.1475-5661.2010.00404.x

Rey, S. \& Janikas, M. (2005). Regional convergence, inequality and space. Journal of Economic Geography, 5, 155-176

Rey, S. \& Montouri, B. (1999). US regional income convergence: a spatial econometric perspective. Regional Studies, 33(2), 143-156. doi: 10.1080/00343409950122945

Sala-i-Martin, X. (1996). The classical approach to convergence analysis. The Economic Journal, 106, 1019-1036. En http://www.nes.ru/dataupload/files/science/reset/Sala_i_Martin1996ej.pdf

Sastré-Gutiérrez, M. \& Rey, S. (2008). Polarización espacial y dinámicas de la desigualdad interregional en México. Problemas del Desarrollo, 39(155), 181-204.

Sen, A. (1992). Inequality re-examined. Oxford: Oxford University Press.

Seth, S. (2009). Inequality, interactions and human development [Special Issue: Selected papers from the 2008 International Conference of the Human Development and Capability Association]. Journal of Human Development and Capabilities, 10(3), 375-396. doi: $10.1080 / 19452820903048878$

Smyth, F. (2008). Medical geography: understanding health inequalities. Progress in Human Geography, 32(1), 119-127.

Székely, M., López-Calva, L., Meléndez, A., Rascón, E. \& Rodríguez-Chamussy, L. (2007). Poniendo a la pobreza de ingresos y a la desigualdad en el mapa de México. Economía Mexicana Nueva Época 16 (2, segundo semestre), 239-303. En http://www.economiamexicana.cide.edu/ num_anteriores/XVI-2/03_SZEKELY.pdf

United Nations Development Programme (UNDP). (2007). Human Development Report 20072008. Fighting climate change: Human solidarity in a divided world. Nueva York: Autor.

World Bank (2005). La pobreza urbana en México. En Generación de ingresos y protección social para los pobres. Informe ejecutivo (pp. 53-66). Washington: Banco Internacional de Reconstrucción y Fomento/Banco Mundial. En http://siteresources.worldbank.org/INTMEXICO/Resources/La_Pobreza_Urbana_en_Mexico.pdf

Ziccardi, A. (2008). Pobreza y exclusión social en las ciudades del siglo XXI. En A. Ziccardi (Comp.), Procesos de urbanización de la pobreza y nuevas formas de exclusión social. Los retos de las politicas sociales de las ciudades latinoamericanas del siglo XXI (pp. 9-33). Bogotá: Siglo del Hombre Editores/Consejo Latinoamericano de Ciencias Sociales (Clacso)-Comparative Research Programme on Poverty (CROP). En http://bibliotecavirtual.clacso.org.ar/ar/ libros/clacso/crop/ziccardi/ 